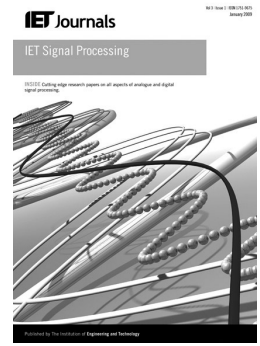

\title{
Interference cancellation beamforming robust to pointing errors
}

\author{
Jie Zhuang, Athanassios Manikas \\ Department of Electrical and Electronic Engineering, Imperial College, London SW7 2AZ, UK \\ E-mail: a.manikas@imperial.ac.uk
}

\begin{abstract}
The conventional Wiener-Hopf beamformer is subject to substantial performance degradation in the presence of steering vector pointing errors. By removing the effects of the desired signal, the modified Wiener-Hopf beamformer avoids this problem but allows cochannel interferences to pass through in order to maximise the signal-to-noise ratio. In this study, a novel array beamformer is proposed, which not only reduces the effect of pointing errors, but also asymptotically provides complete interference rejection. In particular, the proposed beamformer utilises a vector space projection method and employs a one-step computation for the desired signal power. Using this, the effects of the desired signal can be extracted to form the desired-signal-absent covariance matrix. Thus, a weight vector orthogonal with the interference subspace can be constructed. Numerical results demonstrate the superior performance of the proposed beamformer in the presence of pointing errors relative to other existing approaches such as 'diagonal loading', 'robust Capon' and 'signal subspace projection' beamformers.
\end{abstract}

\section{Notation}

$a$
$\underline{A}, \underline{a}$
$(\cdot)^{\mathrm{T}}(\cdot)^{\mathrm{H}}$
$(\cdot)^{*}$
$\mathbb{P}$
$\mathbb{P}^{\perp}$
$\mathbb{V}_{N}$
$\|\cdot\|$
$\mathcal{E}\{\cdot\}$
$\mathcal{P}\{\mathbb{A}\}$
$\operatorname{eig}_{\max }(\mathbb{A})$
$\operatorname{eig}_{i}(\mathbb{A})$
$\mathcal{L}[\mathbb{A}]$

\section{Introduction}

An important topic in array signal processing is interference (e.g. jammers) rejection. This can be achieved using a beamformer which should place relatively high gain in the direction of the desired signal and nulls in the directions of the interferences.

The Wiener-Hopf beamformer is a representative example of a conventional but optimum steering vector beamformer in terms of signal-to-noise-plus-interference ratio (SNIR). However, this scheme compromises on interference suppression, allowing some interferences to pass through (contaminating the desired signal) in order to maximise the signal-to-noise ratio (SNR). The Wiener-Hopf beamformer is also vulnerable to steering vector pointing errors [1], which will cause a substantial degradation in its SNIR performance when the assumed (or nominal) directionof-arrival (DOA) of the desired signal is different from the actual one. By eliminating the effects of the desired signal, the 'modified' Wiener-Hopf beamformer removes this sensitivity to pointing errors [1] but allows interference to pass through as well. Hence, both the Wiener-Hopf beamformer and its modified version fail to provide complete suppression of unwanted interferences.

An alternative approach to design robust beamformers is to apply so-called diagonal loading (DL) [2] or its generalised versions [3-6] to conventional beamformers. However, there is no systematic approach to choose the loading factor for the conventional DL. Instead, it is usually set in an ad hoc way, typically at ten times the noise power. In the robust Capon beamformer (RCB) developed in [4], the DL factor is related to the uncertainty level and can be calculated by solving an optimisation problem where the Lagrange multiplier methodology is utilised. This approach provides an equivalent solution to that in $[5,6]$ where convex optimisation techniques are utilised. However, in this case the optimal solution corresponds to the vector which intends to be close to the principal eigenvector of the received signal covariance matrix as much as the constraint allows [7]. This implies that the estimated manifold will definitely be affected by the interference signals because the principal eigenvector is a linear combination of all the manifold vectors (both the desired and the interferences). As a result it does not provide complete interference rejection.

In [8], a signal-subspace projection (SSP) method is proposed for mitigating the problem of pointing errors. However, this is not an interference cancellation technique 
and the error component lying in the signal subspace cannot be eliminated.

Eigenspace-based interference cancellers, proposed in [9-11], attempt to remove the effects of the desired signals by using a blocking matrix. Here the signals received by adjacent sensors are subtracted, which reduces the dimensionality in observation space. In turn, this implies a reduction in the resolution performance of the array system. Furthermore, the approaches are strictly limited to uniform linear arrays (ULA). In the presence of pointing errors, the desired signal will leak into the output of the blocking matrix, which may result in the severe performance degradation since the desired signal would be contained in the interference subspace and therefore be suppressed instead of being enhanced.

In this paper, a vector space projections (VSP) approach is employed, which allows most of the pointing errors in the manifold vector of the desired signal to be eliminated. The power of the desired signal can be computed in one step using the theory of covariance fitting. The estimates of manifold and power of the desired signal are then utilised to construct the proposed estimate-and-subtract interference cancellation beamformer. In the absence of pointing errors, the proposed beamformer can completely cancel the interferences at the cost of a degradation in output SNR .

The rest of this paper is organised as follows. The WienerHopf, modified Wiener-Hopf arrays and some existing robust beamformers are reviewed in Section 2. The proposed method of interference cancellation is elaborated in Section 3. Simulation results to illustrate the behaviour of the proposed algorithm are presented in Section 4. Finally, the paper is concluded in Section 5.

\section{Problem formulation - optimum steering vector beamformers}

Consider an array of $N$ sensors receiving $M+1$ uncorrelated narrowband signals (one desired signal and $M$ interferences) located in the far-field of the array. The $N \times 1$ snapshot vector $\underline{x}(t)$, received by the array at time $t$, can be modelled as

$$
\underline{x}(t)=m_{\mathrm{d}}(t) \underline{S}\left(\theta_{\mathrm{d}}\right)+\sum_{i=1}^{M} m_{\mathrm{i}}(t) \underline{S}\left(\theta_{\mathrm{i}}\right)+\underline{n}(t)
$$

Here the vectors $\underline{S}\left(\theta_{\mathrm{d}}\right)$ and $\underline{S}\left(\theta_{\mathrm{i}}\right)$ represent the manifold vectors associated with the desired signal and the $i$ th interference, respectively, which are the array response at the directions of $\theta_{\mathrm{d}}$ and $\theta_{\mathrm{i}}$. Furthermore, in (1), $m_{\mathrm{d}}(t)$ and $m_{\mathrm{i}}(t)$ denote the complex envelopes of the desired signal and the $i$ th interference. The vector $\underline{n}(t)$ represents the baseband additive white Gaussian noise with power $\sigma_{\mathrm{n}}^{2}$. The second-order statistics of the $N \times 1$ signal-vector $\underline{x}(t)$ are represented by the covariance matrix $\mathbb{R}_{x x}$ as

$$
\begin{aligned}
\mathbb{R}_{x x} & =\mathcal{E}\left\{\underline{x}(t) \underline{x}^{\mathrm{H}}(t)\right\} \\
& =\sigma_{\mathrm{d}}^{2} \underline{S}\left(\theta_{\mathrm{d}}\right) \underline{S}^{\mathrm{H}}\left(\theta_{\mathrm{d}}\right)+\underbrace{\sum_{i=1}^{M} \sigma_{i}^{2} \underline{S}\left(\theta_{i}\right) \underline{S}^{\mathrm{H}}\left(\theta_{i}\right)+\sigma_{n}^{2} \rrbracket_{N}}_{\underline{\Delta} \mathbb{R}_{i+n}}
\end{aligned}
$$

where $\sigma_{\mathrm{d}}^{2}=\mathcal{E}\left\{m_{\mathrm{d}}(t) m_{\mathrm{d}}^{*}(t)\right\}$ and $\sigma_{i}^{2}=\mathcal{E}\left\{m_{i}(t) m_{i}^{*}(t)\right\}$ denote the powers of the desired signal and the $i$ th interference. Here the matrix $\mathbb{R}_{i+n}$ denotes the covariance matrix without any contribution from the desired signal. In practical applications, the covariance matrix $\mathbb{R}_{x x}$ can be constructed by using $L$ received snapshots $\left\{\underline{x}\left(t_{l}\right), l=1, \ldots, L\right\}$ as follows

$$
\widehat{\mathbb{R}}_{x x}=\frac{1}{L} \sum_{l=1}^{L} \underline{x}\left(t_{l}\right) \underline{x}^{\mathrm{H}}\left(t_{l}\right)
$$

By weighting the array with the vector $\underline{w}$, that is, $y_{\text {out }}(t)=\underline{w}^{\mathrm{H}} \underline{x}(t)$, and then maximising the output SNIR of a steering beamformer towards the direction $\theta_{\mathrm{d}}$ of the desired signal, the Wiener-Hopf optimum solution is derived

$$
\underline{w}_{\mathrm{WH}}=\beta \mathbb{R}_{x x}^{-1} \underline{S}\left(\theta_{\mathrm{d}}\right)
$$

where $\beta$ is a complex constant that does not affect the array output SNIR. When $\beta$ is chosen such that the array gain in the desired direction $\theta_{\mathrm{d}}$ is unity (i.e. $\left|\underline{w}_{\mathrm{WH}}^{\mathrm{H}} \underline{S}\left(\theta_{\mathrm{d}}\right)\right|=1$ ), the Wiener-Hopf beamformer becomes the Capon or minimum variance distortionless response beamformer. Maximising the SNIR can also be achieved by solving the following optimisation problem

$$
\mathrm{SNIR}_{\max }=\max _{\underline{w}} \frac{\sigma_{\mathrm{d}}^{2} \underline{w}^{\mathrm{H}} \underline{S}\left(\theta_{\mathrm{d}}\right) \underline{S}\left(\theta_{\mathrm{d}}\right)^{\mathrm{H}} \underline{w}}{\underline{w}^{\mathrm{H}} \mathbb{R}_{i+n} \underline{w}}
$$

The optimal solution to the above problem is given by

$$
\underline{w}_{m-\mathrm{WH}}=\beta \mathbb{R}_{i+n}^{-1} \underline{S}\left(\theta_{\mathrm{d}}\right)
$$

The beamformer using (6) is called the 'modified' WienerHopf beamformer, which requires the knowledge of the matrix $\mathbb{R}_{i+n}$. Inserting (2) into (4) and using the matrix inversion lemma [12], (4) becomes

$$
\begin{aligned}
\underline{w}_{\mathrm{WH}} & =\beta \mathbb{R}_{x x}^{-1} \underline{S}\left(\theta_{\mathrm{d}}\right) \\
& =\beta\left(\mathbb{R}_{i+n}+\sigma_{\mathrm{d}}^{2} \underline{S}\left(\theta_{\mathrm{d}}\right) \underline{S}^{\mathrm{H}}\left(\theta_{\mathrm{d}}\right)\right)^{-1} \underline{S}\left(\theta_{\mathrm{d}}\right) \\
& =\beta\left(\mathbb{R}_{i+n}^{-1}-\frac{\mathbb{R}_{i+n}^{-1} \sigma_{\mathrm{d}}^{2} \underline{S}\left(\theta_{\mathrm{d}}\right) \underline{S}^{\mathrm{H}}\left(\theta_{\mathrm{d}}\right) \mathbb{R}_{i+n}^{-1}}{1+\sigma_{\mathrm{d}}^{2} \underline{S}^{\mathrm{H}}\left(\theta_{\mathrm{d}}\right) \mathbb{R}_{i+n}^{-1} \underline{S}\left(\theta_{\mathrm{d}}\right)}\right) \underline{S}\left(\theta_{\mathrm{d}}\right) \\
& =\underbrace{\beta \frac{1}{1+\sigma_{\mathrm{d}}^{2} \underline{S}^{\mathrm{H}}\left(\theta_{\mathrm{d}}\right) \mathbb{R}_{i+n}^{-1} \underline{S}\left(\theta_{\mathrm{d}}\right)}}_{\text {scalar }} \mathbb{R}_{i+n}^{-1} \underline{S}\left(\theta_{\mathrm{d}}\right)
\end{aligned}
$$

Here (7) shows that the weight vectors of the 'full' and 'modified' Wiener-Hopf beamformers differ only by a scaling factor. As a result they offer identical maximum SNIR at the output in the absence of pointing errors. However, in practical applications the received signals often consist of the desired signal and the interferences simultaneously, meaning that the desired-signal-absent covariance matrix $\mathbb{R}_{i+n}$ is unavailable or difficult to find. Thus, the weight vector actually used is given by

$$
\widehat{\underline{w}}_{\mathrm{WH}}=\beta \widehat{\mathbb{R}}_{x x}^{-1} \underline{U}_{\mathrm{S}}
$$

where the vector $\underline{U}_{\mathrm{s}}=\underline{S}\left(\theta_{0}\right)$ denotes the presumed manifold vector corresponding to the nominal DOA $\theta_{0}$. The SNIR performance of the Wiener-Hopf beamformer may suffer a substantial degradation in the presence of pointing errors, that is, when

$$
\underline{U}_{\mathrm{s}} \neq \underline{S}\left(\theta_{\mathrm{d}}\right)
$$


It is shown in [1] that the output SNIR of the modified Wiener-Hopf is much less sensitive to pointing errors. However, the Wiener-Hopf and modified Wiener-Hopf beamformers both always allow interference to pass through at the output of an array of sensors, thus contaminating the desired signal. For this reason, the weight vectors provided by these beamformers are not appropriate for complete interference cancellation.

In order to address the problem of pointing errors, some robust beamformers have been designed [13-15]. In [13], an angle update $\Delta \theta$ is searched by solving the following optimisation problem in an iterative manner:

$$
\min _{\Delta \theta}\left(\underline{S}\left(\theta_{0}\right)+\Delta \theta \underline{\dot{S}}\left(\theta_{0}\right)\right)^{\mathrm{H}} \mathbb{R}_{x x}^{-1}\left(\underline{S}\left(\theta_{0}\right)+\Delta \theta \underline{\dot{S}}\left(\theta_{0}\right)\right)
$$

where $\underline{S}\left(\theta_{0}\right)$ denotes the first derivative of the array manifold with respect to the nominal DOA $\theta_{0}$. Then the weight vector is constructed by

$$
\underline{w}_{\mathrm{Er}-\mathrm{Ng}}=\beta \mathbb{R}_{x x}^{-1}\left(\underline{S}\left(\theta_{0}\right)+\Delta \theta \underline{\dot{S}}\left(\theta_{0}\right)\right)
$$

The basic idea of this approach is to search an angle in the vicinity of the nominal desired signal DOA which corresponds to the maximum output power. The beamformer proposed in [14] maintains a gain that is no less than unity in a small area around the nominal desired signal direction, while minimising the output power as much as possible. That is

$$
\begin{aligned}
& \underline{w}_{\text {Chen }}=\min _{\underline{w}} \underline{w}^{\mathrm{H}} \mathbb{R}_{x x} \underline{w} \\
& \text { subject to }\left|\underline{w}^{\mathrm{H}} \underline{S}(\theta)\right| \geq 1 \text {, for } \theta_{\text {low }}<\theta<\theta_{\text {up }}
\end{aligned}
$$

where $\theta_{\text {low }}$ and $\theta_{\text {up }}$ are the preselected lower- and upper-angle bounds in which the desired source could lie. The approach in [15] aims to suppress the sidelobe level where the weight vector is given by

$$
\begin{aligned}
& \underline{w}_{\mathrm{SVM}}=\min _{\underline{w}} \underline{w}^{\mathrm{H}} \mathbb{R}_{x x \underline{w}} \\
& \text { subject to }\left|\underline{w}^{\mathrm{H}} \underline{S}(\theta)-1\right| \leq \varepsilon, \text { for }\left|\theta-\theta_{0}\right| \leq \Delta \theta \\
& \left|\underline{w}^{\mathrm{H}} \underline{S}(\theta)\right| \leq \varepsilon, \text { for }\left|\theta-\theta_{0}\right|>\Delta \theta
\end{aligned}
$$

where $\varepsilon$ is a user-defined sidelobe suppressing level. Then the so-called support vector machine technique is used to find the solution of (13). It is worth noting that these three methods have to employ an iterative process that is time consuming. In contrast, the method proposed in this paper has a closed-form solution.

\section{Proposed interference canceller}

\subsection{Pointing error model}

Using the Taylor expansion and retaining the terms up to the second order, the true array manifold vector can be approximated by

$$
\underline{S}\left(\theta_{\mathrm{d}}\right) \simeq \underline{S}\left(\theta_{0}\right)+\left(\theta_{\mathrm{d}}-\theta_{0}\right) \underline{\dot{S}}\left(\theta_{0}\right)+\frac{\left(\theta_{\mathrm{d}}-\theta_{0}\right)^{2}}{2} \underline{\ddot{S}}\left(\theta_{0}\right)
$$

where $\underline{\tilde{S}}\left(\theta_{0}\right)$ denotes the second derivative of the array manifold vector with respect to the nominal DOA $\theta_{0}$. Note that the idea of Taylor series expansion approximation for the array manifold vector under a source DOA error has also been considered in $[13,16,17]$. Furthermore

$$
\begin{aligned}
& \underline{S}\left(\theta_{0}\right)=\underline{S}\left(\theta_{\mathrm{d}}-\Delta \theta_{\mathrm{d}}\right) \\
& \simeq \underline{S}\left(\theta_{\mathrm{d}}\right)-\Delta \theta_{\mathrm{d}} \underline{\dot{S}}\left(\theta_{\mathrm{d}}\right)+\frac{\left(\Delta \theta_{\mathrm{d}}\right)^{2}}{2} \underline{\ddot{S}}\left(\theta_{\mathrm{d}}\right) \\
& \underline{S}\left(\theta_{0} \pm \Delta \theta\right)=\underline{S}\left(\theta_{\mathrm{d}} \pm \Delta \theta-\Delta \theta_{\mathrm{d}}\right) \\
& \simeq \underline{S}\left(\theta_{\mathrm{d}}\right)+\left( \pm \Delta \theta-\Delta \theta_{\mathrm{d}}\right) \underline{\dot{S}}\left(\theta_{\mathrm{d}}\right) \\
& \quad+\frac{\left( \pm \Delta \theta-\Delta \theta_{\mathrm{d}}\right)^{2}}{2} \underline{\tilde{S}}\left(\theta_{\mathrm{d}}\right)
\end{aligned}
$$

where $\Delta \theta_{\mathrm{d}}=\theta_{\mathrm{d}}-\theta_{0}$ is unknown, but $\Delta \theta$ (which is the expected range of pointing errors) can still be assigned a conservative value. Now $\underline{S}\left(\theta_{\mathrm{d}}\right)$ may be rewritten as

$$
\begin{aligned}
\underline{S}\left(\theta_{\mathrm{d}}\right) \simeq \frac{(\Delta \theta)^{2}-\left(\Delta \theta_{\mathrm{d}}\right)^{2}}{(\Delta \theta)^{2}} \underline{S}\left(\theta_{0}\right) \\
+\frac{\left(\Delta \theta_{\mathrm{d}}\right)^{2}+\Delta \theta \Delta \theta_{\mathrm{d}}}{2(\Delta \theta)^{2}} \underline{S}\left(\theta_{0}+\Delta \theta\right) \\
+\frac{\left(\Delta \theta_{\mathrm{d}}\right)^{2}-\Delta \theta \Delta \theta_{\mathrm{d}}}{2(\Delta \theta)^{2}} \underline{S}\left(\theta_{0}-\Delta \theta\right)
\end{aligned}
$$

Hence, $\underline{S}\left(\theta_{\mathrm{d}}\right)$ belongs to the known linear subspace $\mathcal{L}[\mathbb{H}]$ where

$$
\mathbb{H}=\left[\underline{S}\left(\theta_{0}\right), \quad \underline{S}\left(\theta_{0}-\Delta \theta\right), \quad \underline{S}\left(\theta_{0}+\Delta \theta\right)\right]
$$

It is clear that $\mathbb{H}$ can be easily computed prior to the beamforming processing since $\theta_{0}$ and $\Delta \theta$ are known values.

\subsection{Pointing error reduction using vector subspace projections}

Performing eigendecomposition on $\mathbb{R}_{x x}$ yields

$$
\mathbb{R}_{x x}=\sum_{i=1}^{N} \lambda_{i} \underline{E}_{i} \underline{E}_{i}^{\mathrm{H}}=\mathbb{E}_{\mathrm{s}}\left(\mathbb{D}_{\mathrm{s}}+\sigma_{\mathrm{n}}^{2} \rrbracket_{M+1}\right) \mathbb{E}_{\mathrm{s}}^{\mathrm{H}}+\sigma_{\mathrm{n}}^{2} \mathbb{E}_{\mathrm{n}} \mathbb{E}_{\mathrm{n}}^{\mathrm{H}}
$$

where $\left\{\lambda_{i}, i=1, \ldots, N\right\}$ are the eigenvalues of $\mathbb{R}_{x x}$ arranged in decreasing order (i.e. $\lambda_{1} \geq \ldots \geq \lambda_{N}$ ), $\underline{E}_{i}$ is the eigenvector associated with $\lambda_{i}, \mathbb{E}_{\mathrm{s}}$ and $\mathbb{E}_{\mathrm{n}}$ contain the $M+1$ dominant eigenvectors and the remaining eigenvectors, respectively, that is

$$
\begin{aligned}
\mathbb{E}_{\mathrm{s}} & =\left[\underline{E}_{1}, \underline{E}_{2}, \ldots, \underline{E}_{M+1}\right] \in \mathcal{C}^{N \times(M+1)} \\
\mathbb{E}_{\mathrm{n}} & =\left[\underline{E}_{M+2}, \ldots, \underline{E}_{N}\right] \in \mathcal{C}^{N \times(N-M-1)}
\end{aligned}
$$

and

$$
\mathbb{D}_{\mathrm{s}}=\operatorname{diag}\left\{\lambda_{1}-\sigma_{\mathrm{n}}^{2}, \lambda_{2}-\sigma_{\mathrm{n}}^{2}, \ldots, \lambda_{M+1}-\sigma_{\mathrm{n}}^{2}\right\}
$$

is a diagonal matrix. It is well known that the manifold vector of the desired signal lies in the subspace spanned by the columns of $\mathbb{E}_{\mathrm{s}}$. Now, two constraints $\mathcal{C}_{1}$ and $\mathcal{C}_{2}$ can be 
imposed on $\underline{S}\left(\theta_{\mathrm{d}}\right)$

$$
\begin{aligned}
& \mathcal{C}_{1}=\left\{\underline{S}\left(\theta_{\mathrm{d}}\right): \underline{S}\left(\theta_{\mathrm{d}}\right) \in \mathcal{L}\left[\mathbb{E}_{\mathrm{s}}\right]\right\} \\
& \mathcal{C}_{2}=\left\{\underline{S}\left(\theta_{\mathrm{d}}\right): \underline{S}\left(\theta_{\mathrm{d}}\right) \in \mathcal{L}[\mathbb{H}]\right\}
\end{aligned}
$$

The above implies that the unknown manifold $\underline{S}\left(\theta_{\mathrm{d}}\right)$ can be treated as a vector (or a point) lying within the intersection of $\mathcal{C}_{0} \triangleq \mathcal{C}_{1} \cap \mathcal{C}_{2}$. In accordance with the theorem of sequential VSP [18, 19], the intersection of the two constraint sets can be found by applying the alternating projection algorithm. More specifically, with the start point $\underline{S}\left(\theta_{0}\right)$ the sequence $\left\{\underline{S}_{k}\right\}$ generated by

$$
\underline{S}_{k+1}=\mathbb{P}_{\mathcal{C}_{2}} \mathbb{P}_{\mathcal{C}_{1}} \underline{S}_{k}
$$

will converge to $\underline{S}\left(\theta_{\mathrm{d}}\right)$ as $k \rightarrow \infty$, where the projection operations are defined as

$$
\begin{gathered}
\mathbb{P}_{\mathcal{C}_{1}}=\mathbb{E}_{\mathrm{s}} \mathbb{E}_{\mathrm{s}}^{\mathrm{H}} \\
\mathbb{P}_{\mathcal{C}_{2}}=\mathbb{U}\left(\mathbb{H}^{\mathrm{H}} \mathfrak{H}\right)^{-1} \mathfrak{H}^{\mathrm{H}}
\end{gathered}
$$

However, this requires an iterative process. A solution requiring only one step is now derived. Suppose $\underline{S}_{k} \rightarrow \underline{S}_{\infty}$ as $k \rightarrow \infty$; then (22) becomes

$$
\underline{S}_{\infty}=\mathbb{P}_{\mathcal{C}_{2}} \mathbb{P}_{\mathcal{C}_{1}} \underline{S}_{\infty}
$$

The above equation tells us that the final converged estimate $\underline{S}_{\infty}$ is given by the eigenvector (up to a scaling factor) of the matrix $\mathbb{P}_{\mathcal{C}_{2}} \mathbb{P}_{\mathcal{C}_{1}}$ which has a corresponding eigenvalue equal to one. Also, the maximum eigenvalue of the matrix product $\mathbb{P}_{\mathcal{C}_{2}} \mathbb{P}_{\mathcal{C}_{1}}$ is one. In accordance with Corollary 11 of [20], it can be shown that

$$
\begin{aligned}
& \operatorname{eig} \max \left(\mathbb{P}_{\mathcal{C}_{2}} \mathbb{P}_{\mathcal{C}_{1}}\right) \leq \operatorname{eig} \max \left(\mathbb{P}_{\mathcal{C}_{2}}\right) \max _{\underline{u}^{\mathrm{H}} \underline{u}=1} \underline{u}^{\mathrm{H}} \mathbb{P}_{\mathcal{C}_{1}} \underline{u} \\
& =\max _{\underline{u}^{\mathrm{H}} \underline{\underline{u}} \underline{\underline{u}}=1} \frac{\underline{u}^{\mathrm{H}} \mathbb{P}_{\mathcal{C}_{1}} \underline{u}}{\underline{u}^{\mathrm{H}} \underline{u}} \\
& =\operatorname{eig}_{\max }\left(\mathbb{P}_{\mathcal{C}_{1}}\right) \\
& =1
\end{aligned}
$$

where the fact that the maximum eigenvalues of $\mathbb{P}_{\mathcal{C}_{1}}$ and $\mathbb{P}_{\mathcal{C}_{2}}$ are equal to one is used in the above. Hence, if the nominal $\theta_{0}$ and $\mathbb{E}_{\mathrm{s}}$ are given, the steering vector is computed by

$$
\underline{\widehat{U}}_{\mathrm{s}}=\beta \underline{\mathcal{P}}\left\{\mathbb{P}_{\mathcal{C}_{2}} \mathbb{P}_{\mathcal{C}_{1}}\right\}
$$

where $\beta$ is chosen such that $\left\|\widehat{U}_{\mathrm{s}}\right\|^{2}=N$ and $\mathcal{P}\{A\}$ denotes the principal eigenvector of the matrix argument $A$. Equation (26) can be interpreted as selecting a vector located in the linear subspace spanned by the columns of $\mathbb{H}$, which has the smallest angle from the subspace $\mathcal{L}\left[\mathbb{E}_{\mathrm{s}}\right]$.

\subsection{Power estimation}

In [3], the power associated with the desired signal is estimated by using covariance fitting theory as

$$
\sigma_{\mathrm{d}}^{2}=\frac{1}{\underline{S}^{\mathrm{H}}\left(\theta_{\mathrm{d}}\right) \mathbb{R}_{x x}^{-1} \underline{S}\left(\theta_{\mathrm{d}}\right)}
$$

However, the effect of noise on the signal subspace is neglected in the above. This can be seen in the case where the interferences are absent. Then the covariance matrix becomes $\mathbb{R}_{x x}=\sigma_{\mathrm{d}}^{2} \underline{S}\left(\theta_{\mathrm{d}}\right) \underline{S}^{\mathrm{H}}\left(\theta_{\mathrm{d}}\right)+\sigma_{\mathrm{n}}^{2} \rrbracket_{N}$ and its inverse is readily obtained by

$$
\mathbb{R}_{x x}^{-1}=\frac{1}{\sigma_{\mathrm{n}}^{2}} \rrbracket_{N}-\frac{\sigma_{\mathrm{d}}^{2}}{\sigma_{\mathrm{n}}^{2}\left(\sigma_{\mathrm{n}}^{2}+N \sigma_{\mathrm{d}}^{2}\right)} \underline{S}\left(\theta_{\mathrm{d}}\right) \underline{S}^{\mathrm{H}}\left(\theta_{\mathrm{d}}\right)
$$

Then the power estimation using (27) is given by

$$
\widehat{\sigma}_{\mathrm{d}}^{2}=\sigma_{\mathrm{d}}^{2}+\frac{\sigma_{\mathrm{n}}^{2}}{N} \neq \sigma_{\mathrm{d}}^{2}
$$

In this section, we present a one-step power estimation approach in which the effect of noise on the signal subspace is taken into account. The power of the desired signal $\sigma_{\mathrm{d}}^{2}$ is equivalent to the largest possible value of $\sigma^{2}$ which satisfies

$$
\begin{aligned}
& \operatorname{eig}_{i}\left(\mathbb{R}_{x x}-\sigma^{2} \underline{S}\left(\theta_{\mathrm{d}}\right) \underline{S}^{\mathrm{H}}\left(\theta_{\mathrm{d}}\right)\right) \geq \sigma_{\mathrm{n}}^{2} \\
& \Leftrightarrow \operatorname{eig}_{i}\left(\mathbb{R}_{x x}-\sigma_{\mathrm{n}}^{2} \rrbracket_{N}-\sigma^{2} \underline{S}\left(\theta_{\mathrm{d}}\right) \underline{S}^{\mathrm{H}}\left(\theta_{\mathrm{d}}\right)\right) \geq 0 \\
& \Leftrightarrow \operatorname{eig}_{i}\left(\mathbb{E}_{\mathrm{s}}^{\mathrm{H}}\left(\mathbb{R}_{x x}-\sigma_{\mathrm{n}}^{2} \rrbracket_{N}\right) \mathbb{E}_{\mathrm{s}}\right. \\
& \left.-\sigma^{2} \mathbb{E}_{\mathrm{s}}^{\mathrm{H}} \underline{S}\left(\theta_{\mathrm{d}}\right) \underline{S}^{\mathrm{H}}\left(\theta_{\mathrm{d}}\right) \mathbb{E}_{\mathrm{s}}\right) \geq 0 \\
& \Leftrightarrow \operatorname{eig}_{i}\left(\mathbb{D}_{\mathrm{s}}-\sigma^{2} \mathbb{E}_{\mathrm{s}}^{\mathrm{H}} \underline{S}\left(\theta_{\mathrm{d}}\right) \underline{S}^{\mathrm{H}}\left(\theta_{\mathrm{d}}\right) \mathbb{E}_{\mathrm{s}}\right) \geq 0 \\
& \Leftrightarrow \operatorname{eig}_{i}\left(\mathbb{\square}_{M+1}-\sigma^{2} \mathbb{D}_{\mathrm{s}}^{-(1 / 2)} \mathbb{E}_{\mathrm{s}}^{\mathrm{H}} \underline{S}\left(\theta_{\mathrm{d}}\right) \underline{S}^{\mathrm{H}}\left(\theta_{\mathrm{d}}\right) \mathbb{E}_{\mathrm{s}} \mathbb{D}_{\mathrm{s}}^{-(1 / 2)}\right) \geq 0 \\
& \Leftrightarrow 1-\sigma^{2} \underline{S}^{\mathrm{H}}\left(\theta_{\mathrm{d}}\right) \mathbb{E}_{\mathrm{s}} \mathbb{D}_{\mathrm{s}}^{-1} \mathbb{E}_{\mathrm{s}}^{\mathrm{H}} \underline{S}\left(\theta_{\mathrm{d}}\right) \geq 0 \\
& \Leftrightarrow \sigma^{2} \leq \frac{1}{\underline{S}^{\mathrm{H}}\left(\theta_{\mathrm{d}}\right) \mathbb{E}_{\mathrm{s}} \mathbb{D}_{\mathrm{s}}^{-1} \mathbb{E}_{\mathrm{s}}^{\mathrm{H}} \underline{S}\left(\theta_{\mathrm{d}}\right)}
\end{aligned}
$$

where $\mathbb{D}_{\mathrm{s}}^{-1 / 2}$ denotes Hermitian square root of $\mathbb{D}_{\mathrm{s}}^{-1}$. Here the notation $\operatorname{eig}_{i}(\mathbb{A}) \geq a$ means that the $i$ th largest eigenvalue of $\mathbb{A}$ is not less than $a$. In the above derivation, the fact that $\mathbb{D}_{\mathrm{s}}^{-(1 / 2)} \mathbb{E}_{\mathrm{s}}^{\mathrm{H}} \underline{S}\left(\theta_{\mathrm{d}}\right) \underline{S}^{\mathrm{H}}\left(\theta_{\mathrm{d}}\right) \mathbb{E}_{\mathrm{s}}^{-(1 / 2)} \mathbb{D}_{\mathrm{s}}^{-(1 / 2)}$ is a rank-one matrix with the principal eigenvalue $\underline{S}^{\mathrm{H}}\left(\theta_{\mathrm{d}}\right) \mathbb{E}_{\mathrm{s}} \mathbb{D}_{\mathrm{s}}^{-1} \mathbb{E}_{\mathrm{s}}^{\mathrm{H}} \underline{S}\left(\theta_{\mathrm{d}}\right)$ is used. Hence, the power of the desired signal is

$$
\sigma_{\mathrm{d}}^{2}=\frac{1}{\underline{S}^{\mathrm{H}}\left(\theta_{\mathrm{d}}\right) \mathbb{E}_{\mathrm{s}} \mathbb{D}_{\mathrm{s}}^{-1} \mathbb{E}_{\mathrm{s}}^{\mathrm{H}} \underline{S}\left(\theta_{\mathrm{d}}\right)}
$$

Let us examine the interference-absent case again. Now $\mathbb{D}_{\mathrm{s}}$ becomes a scalar equal to $N \sigma_{\mathrm{d}}^{2}$ and $\underline{S}^{\mathrm{H}}\left(\theta_{\mathrm{d}}\right) \mathbb{E}_{\mathrm{s}} \mathbb{E}_{\mathrm{s}}^{\mathrm{H}} \underline{S}\left(\theta_{\mathrm{d}}\right)=$ $\underline{S}^{\mathrm{H}}\left(\theta_{\mathrm{d}}\right) \underline{S}\left(\theta_{\mathrm{d}}\right)=N$. Thus (31) offers us the precise power estimation. Interestingly, McCloud and Scharf [21] (see Lemma III.1 in [21]) propose an equivalent power estimation method from the basis of the oblique projection. 


\subsection{Beamformer construction}

Using the estimated steering vector in (26) and the estimated power associated with the desired signal in (31), a desired-signal-absent covariance matrix can be formed by

$$
\widehat{\mathbb{R}}_{i+n}=\mathbb{R}_{x x}-\widehat{\sigma}_{\mathrm{d}}^{2} \widehat{\widehat{U}}_{\mathrm{s}} \widehat{\widehat{U}}_{\mathrm{s}}^{\mathrm{H}}
$$

where $\widehat{U}_{\mathrm{s}}$ is obtained by (26) and the power $\widehat{\sigma}_{\mathrm{d}}^{2}$ is computed by (31) with $S\left(\theta_{\mathrm{d}}\right)$ replaced by $\widehat{U}_{\mathrm{s}}$. Performing eigendecomposition on $\widehat{\mathbb{R}}_{i+n}$ yields

$$
\begin{aligned}
\widehat{\mathbb{R}}_{i+n} & =\sum_{i=1}^{N} \widehat{\lambda} \widehat{E}_{i} \widehat{E}_{i}^{\mathrm{H}} \\
& =\widehat{\mathbb{E}}_{I} \widehat{\mathbb{D}}_{I} \widehat{\mathbb{E}}_{I}^{\mathrm{H}}+\widehat{\mathbb{E}}_{O} \widehat{\mathbb{D}}_{O} \widehat{\mathbb{E}}_{O}^{\mathrm{H}}
\end{aligned}
$$

where $\left\{\widehat{\lambda}_{i}, i=1, \ldots, N\right\}$ are the eigenvalues of $\widehat{\mathbb{R}}_{i+n}$ arranged in decreasing order and $\widehat{E}_{i}$ is the eigenvector associated with $\widehat{\lambda}_{i}$. In addition, $\widehat{\mathbb{D}}_{I}$ and $\widehat{\mathbb{D}}_{O}$ are diagonal matrices. $\widehat{\mathbb{E}}_{I}$ and $\widehat{\mathbb{E}}_{O}$ consist of the $M$ dominant eigenvectors and the remaining eigenvectors respectively. That is

$$
\begin{gathered}
\widehat{\mathbb{E}}_{I}=\left[\begin{array}{llll}
\widehat{\underline{E}}_{1}, & \widehat{\widehat{E}}_{2}, & \ldots, & \widehat{\underline{E}}_{M}
\end{array}\right] \\
\widehat{\mathbb{E}}_{O}=\left[\begin{array}{llll}
\widehat{\widehat{E}}_{M+1}, & \widehat{E}_{M+2}, & \ldots, & \widehat{\underline{E}}_{N}
\end{array}\right]
\end{gathered}
$$

Now the interference subspace $\mathcal{H}_{I}$ spanned by $\left[\underline{S}\left(\theta_{1}\right), \quad \underline{S}\left(\theta_{2}\right), \ldots, \underline{S}\left(\theta_{M}\right)\right]$ is equivalent to the subspace spanned by $\widehat{\mathbb{E}}_{I}$. Let $\mathbb{P}_{I}^{\perp}$ be the complement projection operator of the interference subspace. Then $\mathbb{P}_{I}^{\perp}$ can be estimated by

$$
\widehat{\mathbb{P}}_{I}^{\perp}=\mathbb{\square}_{N}-\widehat{\mathbb{E}}_{I} \widehat{\mathbb{E}}_{I}^{\mathrm{H}}
$$

Using this, an interference cancellation weight vector can be constructed by projecting the expected steering vector associated with the desired signal onto the complement of the interference subspace as

$$
\underline{w}_{\mathrm{ic}}=\frac{\widehat{\mathbb{P}}_{I}^{\perp} \widehat{\underline{U}}_{\mathrm{s}}}{\sqrt{\underline{\widehat{U}}_{\mathrm{s}}^{\mathrm{H}} \widehat{\mathbb{P}}_{I}^{\perp} \underline{\underline{U}}_{\mathrm{s}}}}
$$

The output jammer power of the array, using the actual DOA of the desired signal, will be zero because $\underline{w}_{\text {ic }}$ is orthogonal with the interference subspace. This implies that the proposed method can completely cancel the interferences. In the absence of pointing errors, the total output power of the array becomes

$$
P_{\text {out }}=\sigma_{\mathrm{d}}^{2} \underline{S}^{\mathrm{H}}\left(\theta_{\mathrm{d}}\right) \widehat{\mathbb{P}}_{I}^{\perp} \underline{S}\left(\theta_{\mathrm{d}}\right)+\sigma_{\mathrm{n}}^{2}
$$

Thus, the SNR at the array output will be

$$
\mathrm{SNR}_{\text {out }}=\frac{\sigma_{\mathrm{d}}^{2}}{\sigma_{\mathrm{n}}^{2}} \underline{S}^{\mathrm{H}}\left(\theta_{\mathrm{d}}\right) \widehat{\mathbb{P}}_{I}^{\perp} \underline{S}\left(\theta_{\mathrm{d}}\right)
$$

The angle $(\psi)$ between $\mathcal{L}\left[\underline{S}\left(\theta_{\mathrm{d}}\right)\right]$ (the linear subspace spanned by the desired signal) and $\mathcal{H}_{I}^{\perp}$ (the linear subspace orthogonal with the interference signals) is defined as

$$
\psi=\arccos \left(\frac{\sqrt{\underline{S}^{\mathrm{H}}\left(\theta_{\mathrm{d}}\right) \mathbb{P}_{I}^{\perp} \underline{S}\left(\theta_{\mathrm{d}}\right)}}{\sqrt{\underline{S}^{\mathrm{H}}\left(\theta_{\mathrm{d}}\right) \underline{S}\left(\theta_{\mathrm{d}}\right)}}\right)
$$

Therefore (38) is equivalent to

$$
\mathrm{SNR}_{\text {out }}=\frac{\sigma_{\mathrm{d}}^{2}}{\sigma_{\mathrm{n}}^{2}} \underline{S}^{\mathrm{H}}\left(\theta_{\mathrm{d}}\right) \underline{S}\left(\theta_{\mathrm{d}}\right) \cos ^{2} \psi=\frac{\sigma_{\mathrm{d}}^{2}}{\sigma_{\mathrm{n}}^{2}} N \cos ^{2} \psi
$$

The above equation shows that the proposed beamformer can completely cancel the interferences at a cost of output SNR reduction, which is a function of the geometry between the desired and interference subspaces represented by the angle $\psi$.

\subsection{Proposed algorithm}

The proposed algorithm can be presented as a series of steps as follows:

Step 1: Calculate the projection operator $\mathbb{P}_{\mathcal{C}_{2}}$ based on (23b) using $\mathbb{H}$.

Step 2: Estimate the received signal covariance matrix $\mathbb{R}_{x x}$ using (3). Find its signal-eigenvectors $\mathbb{E}_{\mathrm{s}}$ and compute the projection operator $\mathbb{P}_{\mathcal{C}_{1}}$ in (23a).

Step 3: Estimate $\widehat{U}_{\mathrm{s}}$ using (26) and $\widehat{\sigma}_{\mathrm{d}}^{2}$ using (31).

Step 4: Estimate the matrix $\widehat{\mathbb{R}}_{i+n}$ using (32).

Step 5: Perform eigen-decomposition on the matrix $\widehat{\mathbb{R}}_{i+n}$. Find the projection operator $\widehat{\mathbb{P}}_{I}^{\perp}$.

Step 6: Compute the weight vector $\underline{w}_{\text {ic }}$ using (36).

Step 7: Weight the inputs of the array with $\underline{w}_{\mathrm{ic}}$.

\section{Simulation studies}

Assume that one desired signal and three interferences impinge on a ULA of $N=10$ isotropic sensors with half-wavelength inter-sensor spacing. The direction of the desired signal is fixed at $\theta_{\mathrm{d}}=90^{\circ}$ in all the following examples. Unless stated otherwise, both the desired signal and the interference signals are assumed to have unity power (i.e. input signal-to-interference ratio (SIR) is 0 $\mathrm{dB}$ ). In addition, the noise power is $\sigma_{\mathrm{n}}^{2}=1$ (i.e. input $\mathrm{SNR}=0 \mathrm{~dB})$. The matrix $\mathbb{W}$ is constructed by $\mathbb{W}=$ $\left[\underline{S}\left(\theta_{0}\right), \quad \underline{S}\left(\theta_{0}-\Delta \theta\right), \quad \underline{S}\left(\theta_{0}+\Delta \theta\right)\right]$ with $\Delta \theta=2^{\circ}$.

Case 1: In the first example, the directions of the interferers are at $60^{\circ}, 62^{\circ}$ and $70^{\circ}$, and the assumed angle of the desired signal (or the nominal angle) is $90^{\circ}$ (i.e. no pointing error in this example). The resulting array patterns when implementing the Wiener-Hopf, Lee-Lee [11], Er-Ng [13] and the proposed approach are shown in Fig. 1. In LeeLee's method, a blocking matrix $\mathbb{B}_{\text {blk }} \in \mathcal{C}^{N \times(N-q)}$ (where $q$ denotes the dimension reduction) is properly designed to remove the desired signal. Then a desired-signal-absent covariance matrix with reduced dimensionality, $\mathbb{R}_{\mathrm{blk}}=$ $\mathbb{B}_{\mathrm{blk}}^{\mathrm{H}} \mathbb{R}_{x x} \mathbb{B}_{\mathrm{blk}} \in \mathcal{C}^{(N-q) \times(N-q)}$, can be obtained where the signal subspace of this matrix is equivalent to the interference subspace. The final weight vector of the Lee-Lee beamformer is given by $\underline{w}_{\text {lee-lee }}=\beta \mathbb{P}_{n} \underline{S}^{(q)}\left(\theta_{0}\right)$, where $\mathbb{P}_{\mathrm{n}}$ is the projection operator onto the noise subspace of $\mathbb{R}_{\mathrm{blk}}$ and $\underline{S}^{(q)}\left(\theta_{0}\right) \in \mathcal{C}^{(N-q) \times 1}$ is composed of the first $(N-q)$ elements of $S\left(\theta_{0}\right)$. In the simulations, $q=1$ is chosen. Note that Lee-Lee's approach is strictly limited to 


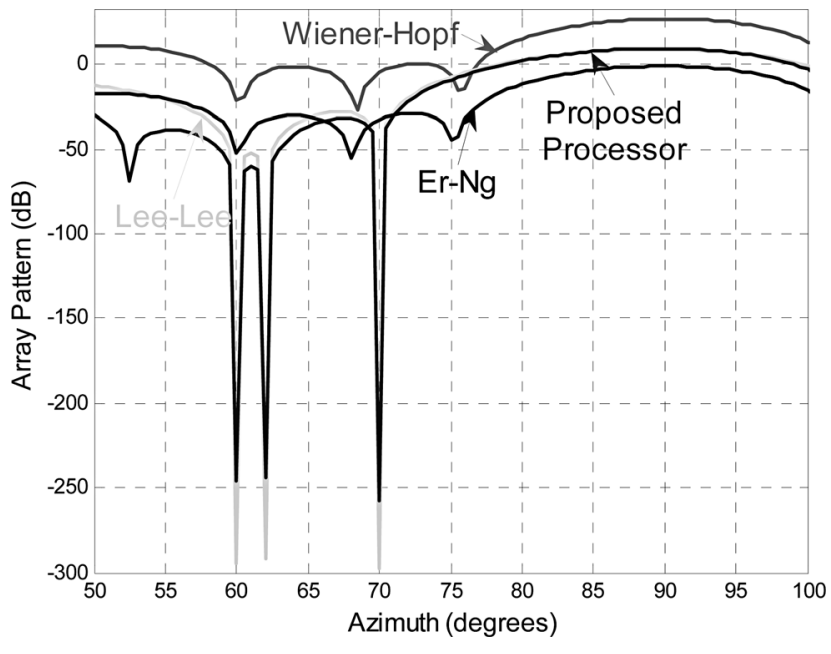

Fig. 1 Array pattern in Case 1 with three interferences at $60^{\circ}, 62^{\circ}$ and $70^{\circ}$

DOA of the desired signal is $90^{\circ}$ and the pointing angle is $90^{\circ}$ as well. Input SNR and input $\mathrm{SIR}=0 \mathrm{~dB}$

ULAs. It can be seen in Fig. 1 that neither the Wiener-Hopf beamformer nor Er-Ng's beamformer can distinguish (resolve) the two closely spaced interfering sources (at $60^{\circ}$ and $62^{\circ}$ ) correctly but this is possible with the beamformer based on the proposed interference cancellation approach in (36) or using Lee-Lee's approach. In addition, both LeeLee's method and the proposed method present very deep nulls at the locations of the unknown interferences, but allow the desired signal to pass through. An added advantage of these two approaches is that each deep null provides the location estimate of an unknown interference. Finally, it can be seen in Fig. 1 that the Wiener-Hopf beamformer and Er$\mathrm{Ng}$ 's beamformer allow the interference to partially pass through because the associated array pattern cannot place deep nulls at the locations of interferences.

The power estimations are listed in Table 1 which illustrate that the proposed method provides the precise estimate, whereas there exists estimation error when using the method proposed in [3].

Case 2: Comparison is now made with respect to pointing error effects in the Wiener-Hopf ('modified' and 'full' version) method, Lee-Lee's method and the proposed approach. The DOAs of interferences are $60^{\circ}, 80^{\circ}$ and $100^{\circ}$, whereas the nominal DOA of the desired signal varies from $85^{\circ}$ to $95^{\circ}$ (i.e. the pointing error changes from $-5^{\circ}$ to $+5^{\circ}$ ). Although Fig. $2 a$ shows that the proposed beamformer is more susceptible to pointing errors than the 'modified' Wiener-Hopf solution, it performs much better than the Lee-Lee and 'full' Wiener-Hopf beamformers. In the presence of pointing errors, the desired signal leaks into the output of the blocking matrix and is suppressed as interference, which is the reason for the severe performance degradation in the Lee-Lee beamformer. The SNIR of Lee-Lee without pointing error is below the optimum SNIR. This is because the dimensionality of the observation space is reduced to construct the blocking matrix.

Table 1 Estimated power of the desired signal

\begin{tabular}{lcc}
\hline Actual power & Proposed & Stoica et al. [3] \\
\hline 1 & 1 & 1.1069 \\
\hline
\end{tabular}

The proposed method is also compared with three other robust beamformers [13-15] which also aim to address the problem of pointing errors. For the beamformer proposed in [14], the parameters $\theta_{\text {low }}=\theta_{0}-2^{\circ}$ and $\theta_{\text {up }}=\theta_{0}+2^{\circ}$ are used. Furthermore, $\Delta \theta=2^{\circ}$ and $\varepsilon=0.001$ are chosen for the
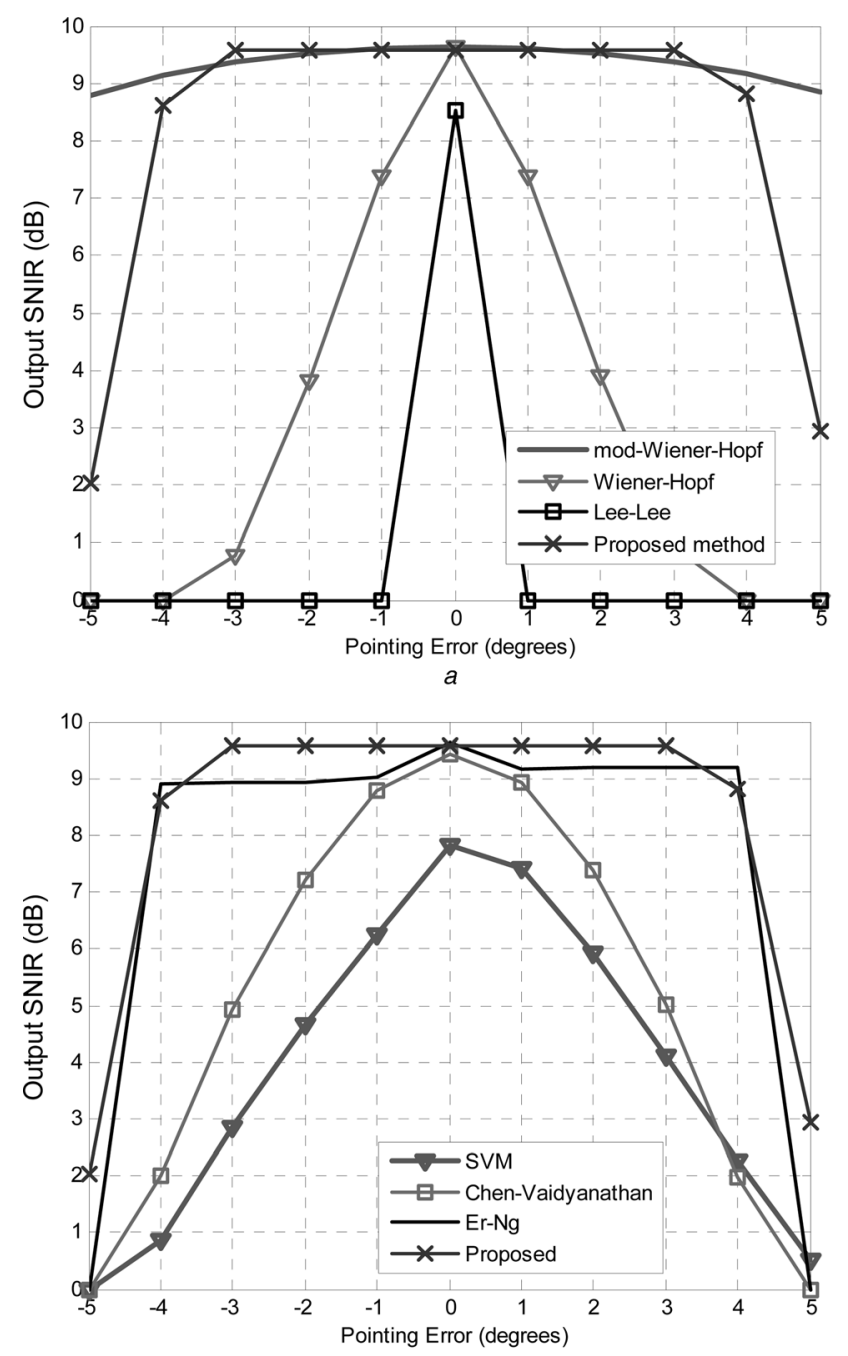

$b$

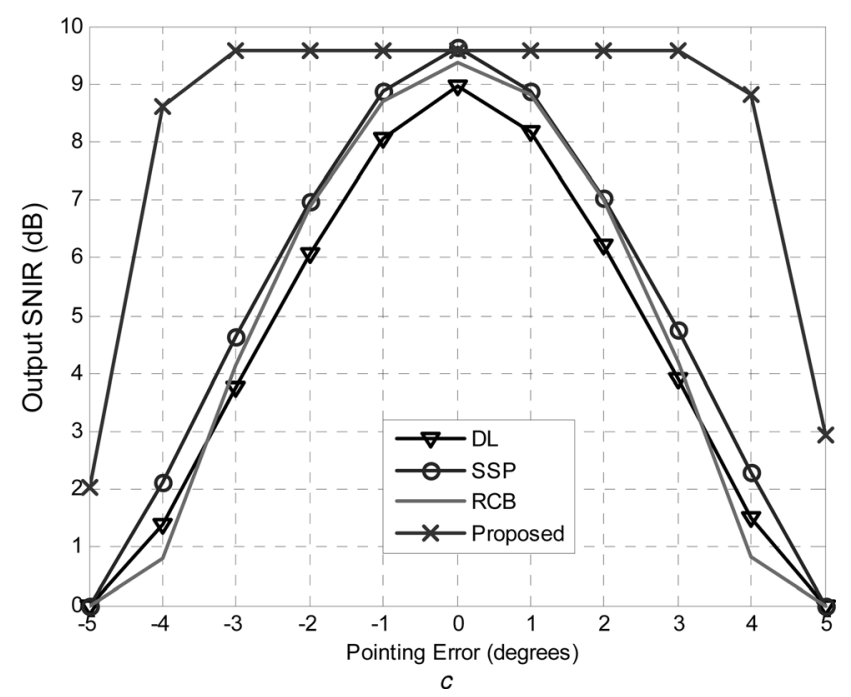

Fig. 2 Effects of pointing errors in Case 2

Nominal direction of the desired signal varies from $85^{\circ}$ to $95^{\circ}$, whereas the true angle remains at $90^{\circ}$. The DOAs of interference signals are $60^{\circ}, 80^{\circ}$ and $100^{\circ}$. Both input SNR and input $\operatorname{SIR}=0 \mathrm{~dB}$ 
beamformer in (13) proposed in [15]. Fig. $2 b$ shows that the proposed beamformer has better output SNIR performance than that in $[13,14]$ or $[15]$.

Three additional popular robust beamformers are also simulated in the same environment and the results are shown in Fig. 2c. The first robust algorithm is the conventional DL [2] with the weight vector given by

$$
\underline{w}_{\mathrm{DL}}=\beta\left(\mathbb{R}_{x x}+\xi \rrbracket_{N}\right)^{-1} \underline{S}\left(\theta_{0}\right)
$$

where the DL factor is $\xi=10 \sigma_{\mathrm{n}}^{2}$. The second beamformer employs the SSP method proposed in [8] and its weight vector is given by

$$
\underline{w}_{\mathrm{SSP}}=\mathbb{R}_{x x}^{-1} \mathbb{P}_{\mathcal{C}_{1}} \underline{S}\left(\theta_{0}\right)
$$

Finally, the RCB presented in [4] is also examined where the ellipsoidal set used is given by

$$
\underline{S}\left(\theta_{\mathrm{d}}\right)=\mathbb{B} \underline{u}+\underline{S}\left(\theta_{0}\right),\|\underline{u}\| \leq 1
$$

with $\quad \mathbb{B}=\left[\underline{S}\left(\theta_{0}\right)-\underline{S}\left(\theta_{0}-\Delta \theta\right), \quad \underline{S}\left(\theta_{0}\right)-\underline{S}\left(\theta_{0}+\Delta \theta\right)\right]$ and $\Delta \theta=2^{\circ}$. Fig. $2 c$ shows that the proposed method
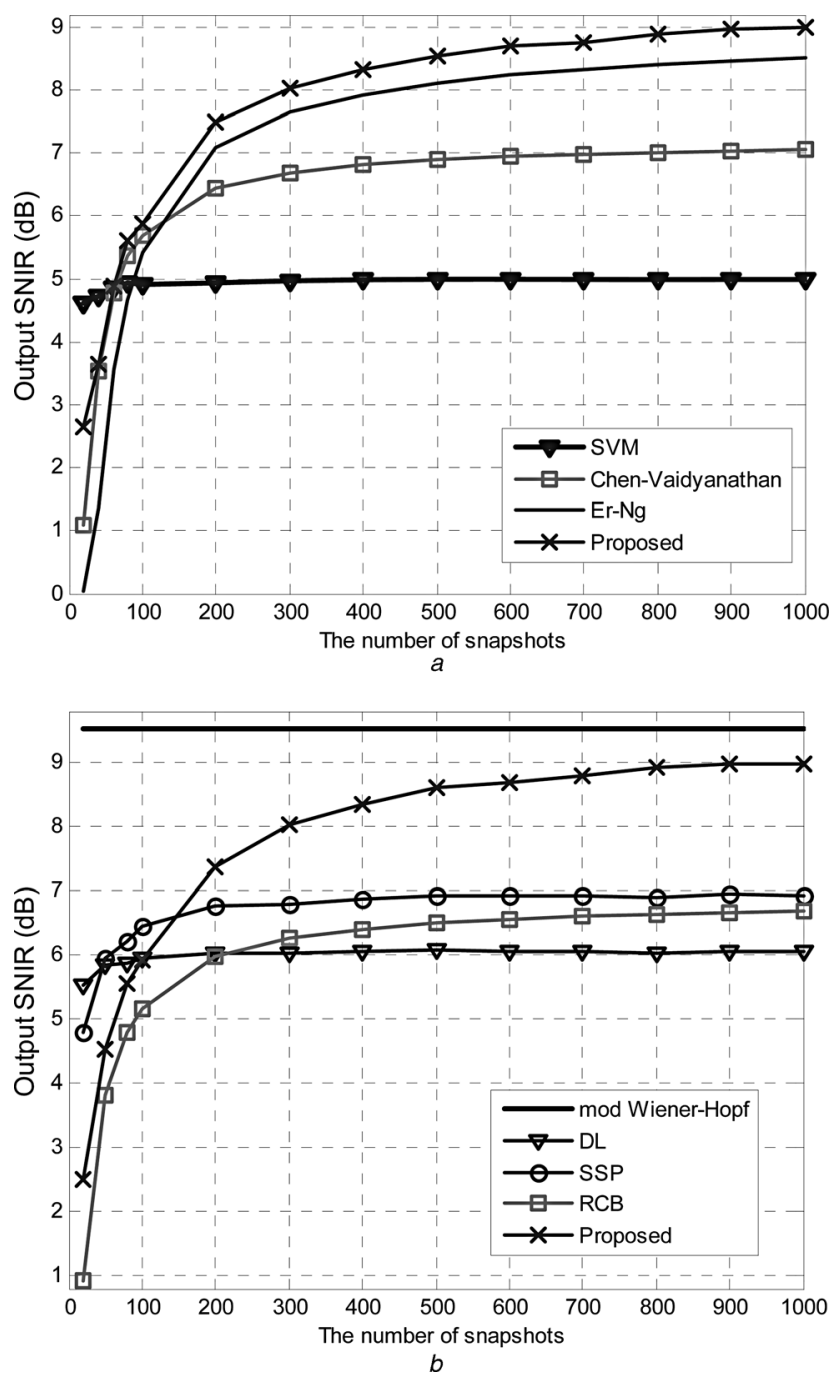

Fig. 3 Effects of a finite number of snapshots in Case 3

True direction of the desired signal is $90^{\circ}$ and the nominal angle is $88^{\circ}$. The DOAs of interference signals are $60^{\circ}, 80^{\circ}$ and $100^{\circ}$. Input SNR and input $\mathrm{SIR}=0 \mathrm{~dB}$ outperforms these three methods in the presence of pointing errors. It can be seen that the RCB cannot provide the optimal SNIR in the absence of pointing errors. This is because the manifold vector used by the RCB always attempts to approach the principal eigenvector of the covariance matrix $\mathbb{R}_{x x}$. However, the principal eigenvector is a linear combination of all the manifold vectors (both the desired and the interferences) [7] and therefore the estimated manifold vector will definitely be affected by the interference signals.

Case 3: The effect of finite snapshots is tested in the third example. The simulation environment is still the same as the second example except that now the nominal direction of the desired signal is fixed at $\theta_{0}=88^{\circ}$ (i.e. $-2^{\circ}$ pointing error). The array output SNIRs against the snapshot number varying from 20 to 1000 are plotted in Fig. 3. This example also indicates that the proposed method is superior to others.

Case 4: In the fourth example, the situation when interferences become dominant is examined. The SIR is decreased from 0 to $-20 \mathrm{~dB}$, and the other simulation parameters are the same as the second example. It can be seen in Fig. 4 that the two subspace-based methods (the proposed method and the SSP method) are robust to the
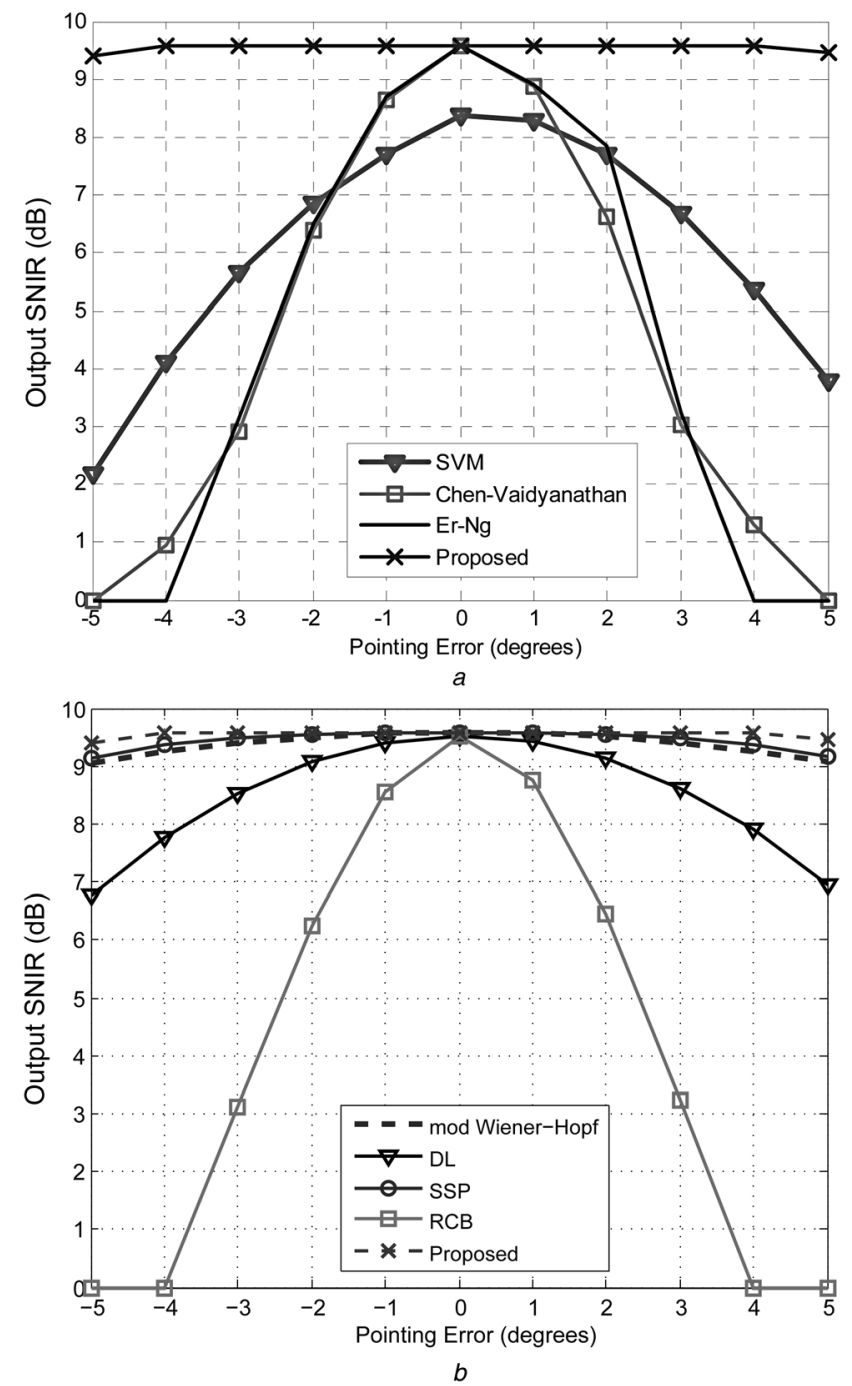

Fig. 4 Effects of pointing errors in Case 4 when the interference signals are dominant with the input $S I R=-20 \mathrm{~dB}$

Nominal direction of the desired signal varies from $85^{\circ}$ to $95^{\circ}$, whereas the true angle maintains at $90^{\circ}$. DOAs of interference signals are $60^{\circ}, 80^{\circ}$ and $100^{\circ}$. Input $\mathrm{SNR}=0 \mathrm{~dB}$ 


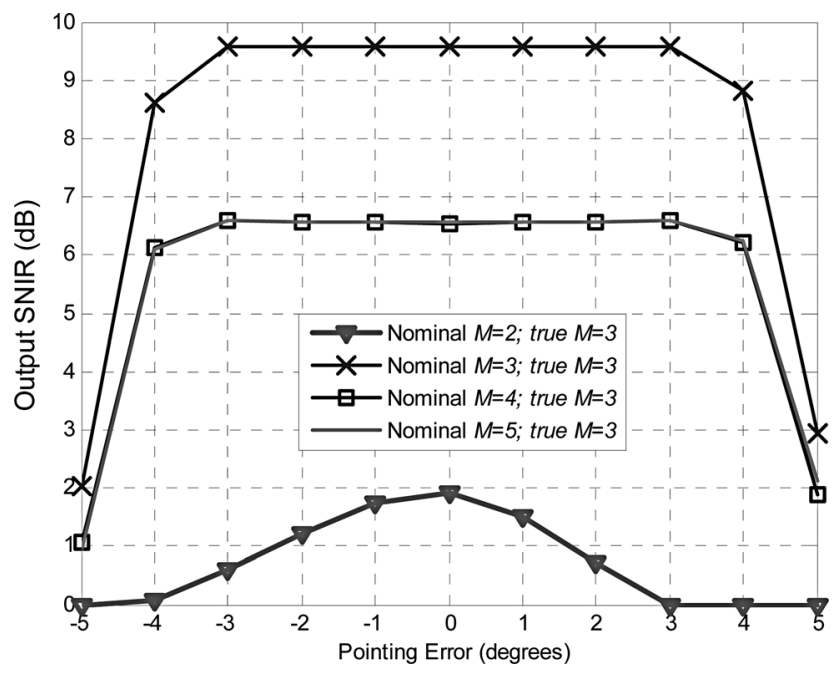

Fig. 5 Effects of misestimation of the interference number $M$ in Case 5

Nominal direction of the desired signal varies from $85^{\circ}$ to $95^{\circ}$, whereas the true angle maintains at $90^{\circ}$. The DOAs of interference signals are $60^{\circ}, 80^{\circ}$ and $100^{\circ}$. Input $\mathrm{SNR}$ and input $\mathrm{SIR}=0 \mathrm{~dB}$

case of dominant interferences. Note that the SNIR performance of Er-Ng's beamformer [13] in Fig. 4a suffers a substantial degradation when the input SIR reduced from 0 to $-20 \mathrm{~dB}$ (see Fig. $2 b$ ). However, there is a reasonable explanation for this. In the optimisation problem of (10), the vector $\underline{S}\left(\theta_{0}\right)+\Delta \theta \underline{\dot{S}}\left(\theta_{0}\right)$ corresponding to the optimum solution of (10) tends towards the principal eigenvector of $\mathbb{R}_{x x}$. In the presence of dominant interference signals, however, the principal eigenvector of $\mathbb{R}_{x x}$ contains significant contributions because of the interference signals. This implies that the vector $\underline{S}\left(\theta_{0}\right)+\Delta \theta \underline{\dot{S}}\left(\theta_{0}\right)$ used in (11) to construct Er-Ng's weight vector will approach the interference signals nearby rather than the desired signal. Consequently, Er-Ng's output SNIR performance is reduced in this example.

Case 5: The situation where the number of signals is estimated incorrectly is examined in the final example. The simulation environment is the same as the second example, except the assumed number of interference varies from 2 to 5 (where the actual number of interference is 3 ). Fig. 5 displays that the proposed method provides acceptable SNIR performance when the number of signals is overestimated, whereas it almost fails to work in the case that the number of signals is underestimated. This can be explained by the following fact. When the signal number is overestimated, the dimension of the signal subspace $\mathcal{L}\left[\mathbb{E}_{\mathrm{s}}\right]$ is increased. However, $\underline{S}\left(\theta_{\mathrm{d}}\right) \subset \mathcal{L}\left[\mathbb{E}_{\mathrm{s}}\right]$ still holds. Hence, the derivation using VSP is still valid. Nevertheless, underestimating the number of signals leads to $\underline{S}\left(\theta_{\mathrm{d}}\right)$ not being completely contained in the subspace $\mathcal{L}\left[\mathbb{E}_{\mathrm{s}}\right]$ and consequently the performance is degraded.

\section{Conclusion}

The true manifold vector of the desired signal differs from the nominal one when pointing errors occur. However, the actual manifold vector can be assumed to lie in a linear subspace which can be obtained easily prior to the beamforming processing using a Taylor series expansion around the nominal DOA. Moreover, the true manifold vector also belongs to the signal subspace which can be found by performing eigen-decomposition on the covariance matrix of the received array data. Using these properties, the estimate of the desired signal manifold vector can be obtained using VSP which eliminates much of the pointing error. In addition, the power of the desired signal can be computed in a one-step operation using the theory of covariance fitting. The estimates of the manifold vector corresponding to the desired signal and its power are then used to construct an estimate-and-subtract interference cancellation beamformer. In the absence of pointing errors, the proposed beamformer can completely cancel the interferences with the output of the array consisting only of desired signal and thermal noise.

\section{References}

1 Cox, H.: 'Resolving power and sensitivity to mismatch of optimum array processors', J. Acoust. Soc. Am., 1973, 54, (3), pp. 771-785

2 Cox, H., Zeskind, R., Owen, M.: 'Robust adaptive beamforming', IEEE Trans. Acoust. Speech Signal Process., 1987, 35, (10), pp. 1365-1376

3 Stoica, P., Wang, Z., Li, J.: 'Robust Capon beamforming', IEEE Signal Process. Lett., 2003, 10, (6), pp. 172-175

4 Li, J., Stoica, P., Wang, Z.: 'On robust Capon beamforming and diagonal loading', IEEE Trans. Signal Process., 2003, 51, (7), pp. $1702-1715$

5 Vorobyov, S., Gershman, A., Luo, Z.-Q.: 'Robust adaptive beamforming using worst-case performance optimization: a solution to the signal mismatch problem', IEEE Trans. Signal Process., 2003, 51, (2), pp. 313-324

6 Lorenz, R., Boyd, S.: 'Robust minimum variance beamforming', IEEE Trans. Signal Process., 2005, 53, (5), pp. 1684-1696

7 Rubsamen, M., Gershman, A.B.: 'Robust adaptive beamforming using multidimensional covariance fitting', IEEE Trans. Signal Process., 2012, 60, pp. 740-753

8 Feldman, D.D., Griffiths, L.J.: 'A projection approach for robust adaptive beamforming', IEEE Trans. Signal Process., 1994, 42, (4), pp. $867-876$

9 Citron, T., Kailath, T.: 'An improved eigenvector beamformer'. IEEE Int. Conf. on Acoust. Speech Signal Process. (ICASSP), 1984, vol. 9, pp. 718-721

10 Haimovich, A., Bar-Ness, Y.: 'An eigenanalysis interference canceler', IEEE Trans. Signal Process., 1991, 39, (1), pp. 76-84

11 Lee, J.-H., Lee, C.-C.: 'Analysis of the performance and sensitivity of an eigenspace-based interference canceler', IEEE Trans. Antennas Propag., 2000, 48, (5), pp. 826-835

12 Golub, G.H., Van Loan, C.F.: 'Matrix computations', (The Johns Hopkins University Press, October 1996)

13 Er, M.H., Ng, B.C.: 'A new approach to robust beamforming in the presence of steering vector errors', IEEE Trans. Signal Process., 1994, 42, (7), pp. 1826-1829

14 Chen, C.-Y., Vaidyanathan, P.P.: 'Quadratically constrained beamforming robust against direction-of-arrival mismatch', IEEE Trans. Signal Process., 2007, 55, (8), pp. 4139-4150

15 Gaudes, C.C., Santamaria, I., Via, J., Gomez, E.M.M., Paules, T.S. 'Robust array beamforming with sidelobe control using support vector machines', IEEE Trans. Signal Process., 2007, 55, (2), pp. 574-584

16 Besson, O., Scharf, L., Vincent, F.: 'Matched direction detectors and estimators for array processing with subspace steering vector uncertainties', IEEE Trans. Signal Process., 2005, 53, (12), pp. 4453-4463

17 Er, M., Ng, B.: 'A self-steered array system for microwave communications', IEEE Trans. Antennas Propag., 1994, 42, (8), pp. 1192-1195

18 Stark, H., Yang, Y.: 'Vector space projections: a numerical approach to signal and image processing, neural nets, and optics', (John Wiley \& Sons, New York, NY, USA, 1998)

19 Gu, J., Stark, H., Yang, Y.: 'Wide-band smart antenna design using vector space projection methods', IEEE Trans. Antennas Propag., 2004, 52, (12), pp. 3228-3236

20 Zhang, F., Zhang, Q.: 'Eigenvalue inequalities for matrix product', IEEE Trans. Autom. Control, 2006, 51, (9), pp. 1506-1509

21 McCloud, M., Scharf, L.: 'A new subspace identification algorithm for high-resolution DOA estimation', IEEE Trans. Antennas Propag., 2002, 50, (10), pp. 1382-1390 